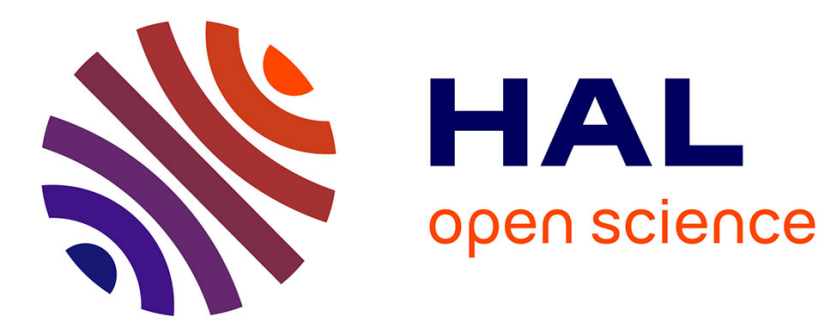

\title{
Vibration source identification using corrected finite difference schemes
}

\author{
Q. Leclere, Charles Pezerat
}

\section{To cite this version:}

Q. Leclere, Charles Pezerat. Vibration source identification using corrected finite difference schemes. Journal of Sound and Vibration, 2012, 331, pp.1366-1377. 10.1016/j.jsv.2011.11.002 . hal-00664285

\section{HAL Id: hal-00664285 \\ https://hal.science/hal-00664285}

Submitted on 29 Mar 2016

HAL is a multi-disciplinary open access archive for the deposit and dissemination of scientific research documents, whether they are published or not. The documents may come from teaching and research institutions in France or abroad, or from public or private research centers.
L'archive ouverte pluridisciplinaire HAL, est destinée au dépôt et à la diffusion de documents scientifiques de niveau recherche, publiés ou non, émanant des établissements d'enseignement et de recherche français ou étrangers, des laboratoires publics ou privés. 


\title{
Vibration source identification using corrected finite difference schemes
}

\author{
Quentin Leclère ${ }^{\mathrm{a}, *}$ Charles Pézerat ${ }^{\mathrm{b}}$ \\ ${ }^{a}$ Laboratoire Vibrations Acoustique, INSA Lyon, 25 bis avenue Jean Capelle \\ F-69621 Villeurbanne Cedex, FRANCE \\ b Laboratoire d'acoustique de l'université du Maine, Université du Maine, CNRS, \\ avenue Olivier Messiaen 72085 Le Mans Cedex 9, FRANCE
}

\begin{abstract}
This paper addresses the problem of the location and identification of vibration excitations from the measurement of the displacement field of a vibrating structure. It constitutes an improvement of the Force Analysis Technique published several years ago. The development is based on the use of the motion equation which is discretized by finite difference schemes approximating spatial derivatives of the displacement. In a first instance, the error due to this approximation is analytically calculated in the case of beams and the low-pass filtering effect of the finite difference schemes in the wavenumber domain is shown. This filter also contains singularities implying systematic errors, especially if the spacing between sensors is chosen in order to regularize the problem. In a second instance, a corrected value is applied to the classic schemes used in the Force Analysis Technique, which suppresses completely the singularities of the filter. After the complete description of the Corrected Force Analysis Technique on beams, the extension to plates is described, where simulations with noisy data show the very good accuracy one can obtain without the
\end{abstract}


requirement of an added regularization for the excitation identification.

Key words: Fuzzy cyclostationarity, synchronous average, angular sampling, instantaneous rotation speed, diesel engine diagnosis, diesel engine noise

\section{Introduction}

The identification of sources acting on structures from operating measurements is a problem that has been widely addressed in the literature, since the pioneer works published in the early eighties $[1,2]$. The most important part of this literature concerns the quantification of a priori localized forces, using the structure's itself as a multi-dimensional force sensor [3]. This approach is also the basis of Transfer Path Analysis [4,5].

The Force Analysis Technique (FAT) is an alternative experimental method which localizes and quantifies vibration sources from the knowledge of the displacement field of a structure and from a local analytical description of the structure's behavior. This method is also known by its acronym RIFF coming from the French language: Résolution Inverse Filtrée Fenêtrée, which means Windowed and Filtered Inverse Resolution. The principle of the FAT is based on the verification of the equation of motion, which describes the equilibrium of internal and external forces applied locally to a little part of the structure. In practice, it consists in injecting measured displacements in the discretized equation of motion, where the spatial derivatives are approximated by finite differences. Because derivatives have the particularity to amplify the noise in

\footnotetext{
* Corresponding author. Fax: 33.4.72.43.87.12. E-mail address: quentin.leclere@insa-lyon.fr.
} 
data, the FAT contains a regularization step, which consists in filtering the informations located in the high wavenumber domain. This filtering is realized by a discrete convolution with a finite response of a low-pass wavenumber filter. During the last decade, the FAT was developed for beams [6], plates [7] and shells [8].

The problem of source localization on thin structures is also addressed in the domain of structural intensity $[9,10]$. The intensity is assessed using either Finite Differences $[10,11]$ or the spatial Finite Fourier Transform [11,12]. The second approach using FFT is generally preferred because of potential errors when using finite difference approximations, and because the measurement noise can be efficiently removed by filtering high wavenumber components. The spatial FFT has been used also for works dealing with source localization on beams [13]. The drawback of using this kind of wavenumber projection is that the response of the structure has to be measured at a large number of points over a wide spatial aperture, even if one wants to assess the load at only few points of the structure.

The principal strength of the FAT is the constitution of an inverse problem using little information from the studied system. Indeed, the verification of the equation of motion is a local view, so it does not require the knowledge of the boundary conditions or the knowledge of the possible sources located outside the studied area, even if they generate vibrations in the whole structure area. The FAT is also particularly appreciated, because it identifies the force distribution, which allows the method to analyze any kind of excitations (forces, moments, pressures, etc. $[7,14]$ ). Today, industrial applications of the FAT concerns essentially the structure-borne noise characterization [15].

Of course, the easiness of the FAT comes from the use of non contact measurement devices, like the scanning laser vibrometer. The recent study coupling 
it with acoustic holography [16] also gives an interesting approach, since the vibration sources can be identified from acoustic measurements. In spite of these advances, the main restraint of the FAT is certainly the uncertain number of measurement it requires. This number is clear when one considers only the finite difference scheme (generally developed at the first order), because it depends only on the order of the spatial derivatives to approximate. However, the use of the low-pass wavenumber filter requires more points and the number of points depends on the cutoff wavenumber to adjust. Then the idea of the required number of points becomes uncertain, because the optimum cutoff wavenumber depends on the frequency and on the level of uncertainties in data $[6,7]$.

This paper proposes an improvement of the FAT, where the use of the filtering by a convolution product is completely suppressed. The idea is to take the advantage of the finite difference scheme which constitutes also a low-pass wavenumber filtering. It is then shown that the use of larger spacing between points is possible and the number of measurements can then be considerably reduced. The paper also gives the required corrections that must be added in the derivative assessments. This new development of FAT is described here for beams (1D structure) and for plates (2D structure). 


\section{Identification of load distributions on beams}

\subsection{Classic FAT for flexural beams}

The equation of motion for flexural beams at the angular frequency $\omega$ is

$$
E I \frac{\partial^{4} w}{\partial x^{4}}-\rho S \omega^{2} w(x)=p(x)
$$

where $p(x)$ is the force distribution in $N / m, w(x)$ is the displacement in $m, E$ is the complex Young's modulus and $I$ is the flexural moment of inertia. The principle of FAT is to directly assess the right hand side member of equation (1), $p(x)$, from the experimental estimation of the left hand side member. To do this, a finite difference approximation is used to approximate the fourth derivative of the displacement:

$$
\frac{\partial^{4} w}{\partial x^{4}} \approx \delta_{\Delta}^{4 x}=\frac{w(x-2 \Delta)-4 w(x-\Delta)+6 w(x)-4 w(x+\Delta)+w(x+2 \Delta)}{\Delta^{4}},
$$

where $\Delta$ is the spacing between two consecutive points of the experimental mesh.

The FAT estimation of the force distribution at a point located at $x$ is thus obtained using eq. (2) in eq. (1):

$$
p^{F A T}(x)=E I \delta_{\Delta}^{4 x}-\rho S \omega^{2} w(x) .
$$

\subsection{Wavenumber response of FAT for flexural beams}

The Fourier transform of equation (1) is

$$
\left(E I k^{4}-\rho S \omega^{2}\right) \hat{w}(k)=\hat{p}(k),
$$


where $\hat{w}(k)$ and $\hat{p}(k)$ indicates the Fourier Transforms of $w(x)$ and $p(x)$. The Fourier Transform of equation (2) is:

$$
\begin{aligned}
\delta_{\Delta}^{\hat{4} x}(k) & =\frac{1}{\Delta^{4}} \hat{w}(k)\left(e^{-2 j k \Delta}-4 e^{-j k \Delta}+6-4 e^{j k \Delta}+e^{2 j k \Delta}\right) \\
& =\hat{w}(k)(2 \cos (2 k \Delta)-8 \cos (k \Delta)+6) / \Delta^{4} \\
& =\hat{w}(k) \kappa_{1 D}^{4},
\end{aligned}
$$

where $\delta_{\Delta}^{\hat{4} x}(k)$ is the Fourier Transform of $\delta_{\Delta}^{4 x}$, defined in eq. 2. The FAT estimation of the force distribution in the wavenumber domain is thus:

$$
\hat{p}^{F A T}(k)=\hat{w}(k)\left(E I \kappa_{1 D}^{4}-\rho S \omega^{2}\right) .
$$

It is now possible to express the ratio between the exact and the identified force distributions in the wavenumber domain:

$$
E^{1 D}(\omega, \Delta, k)=\frac{\hat{p}^{F A T}(k)}{\hat{p}(k)}=\frac{\kappa_{1 D}^{4}-k_{N}^{4}}{k^{4}-k_{N}^{4}}
$$

where $k_{N}$ is the flexural wavenumber of the beam:

$$
k_{N}=\sqrt[4]{\frac{\rho S}{E I} \omega^{2}}
$$

The term expressed in eq. (7) can be considered as the response of FAT in the wavenumber domain. It can also be seen as the systematic error between identified and exact input forces. Considering $n$ the number of points per natural (flexural) wavelength:

$$
n=\frac{\lambda_{N}}{\Delta}=\frac{2 \pi}{k_{N} \Delta}
$$

the systematic error can be written with respect to the non-dimensional parameter $\alpha=k / k_{N}$ :

$$
E^{1 D}(\alpha, n)=\frac{\left(\frac{n}{2 \pi}\right)^{4}\left(2 \cos \left(\frac{4 \pi}{n} \alpha\right)-8 \cos \left(\frac{2 \pi}{n} \alpha\right)+6\right)-1}{\alpha^{4}-1} .
$$


In figure $1, E^{1 D}(\alpha, n)$ is plotted for different values of $n$ (for a given experimental case, $n$ is a continuous variable decreasing with the frequency). It is a low pass filter, with a cut-off wavenumber at $k=k_{N}$ and a cut-off slope increasing when $n$ decreases. The filter is also characterized by two singularities at $k=k_{N}$ and $k=\kappa_{1 D}$, corresponding to roots of the denominator and numerator of equation (7). These singularities overestimate and underestimate strongly the result in narrow wavenumber bands.

The low-pass effect is in fact a real advantage for FAT: the load distribution is identified at discrete points of the structure, and this effect is a welcomed anti-aliasing filter. The Shannon's sampling criterion is written as follows:

$$
\lambda>2 \Delta, \text { or } k<\frac{\pi}{\Delta}
$$

This leads to the following relation between $n$ and $\alpha$ :

$$
\alpha<n / 2
$$

The cut-off wavenumber of ideal anti-aliasing filters are also indicated in figure 1 (the Nyquist wavenumber). The value of FAT responses at these wavenumbers is between -20 and $-15 \mathrm{~dB}$ for $\mathrm{n}$ varying between 3 and 6 .

\subsection{Correction of FAT for flexural beams}

The roots of the numerator of $E^{1 D}$ are solutions of:

$$
\left(2 \cos \left(2 k_{r} \Delta\right)-8 \cos \left(k_{r} \Delta\right)+6\right) / \Delta^{4}-k_{N}^{4}=0,
$$

or, using $X=\cos \left(k_{r} \Delta\right)$ :

$$
\left(4 X^{2}-8 X+4\right) / \Delta^{4}-k_{N}^{4}=0 .
$$




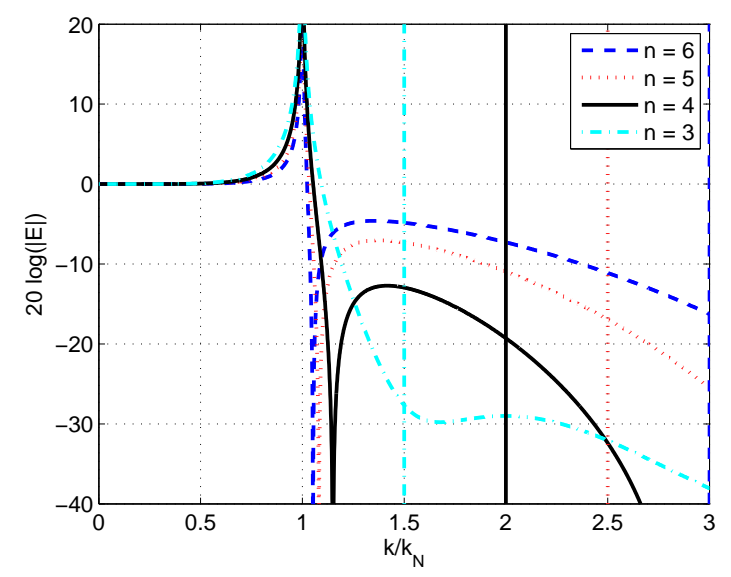

Fig. 1. Response of the classic FAT identification in the wavenumber domain. Vertical lines indicate the Nyquist wavenumbers of the the spatial samplings. This polynomial function has only one root lower than the unity:

$$
X=1-\frac{\Delta^{2} k_{N}^{2}}{2} .
$$

The solutions of eq. (9) are thus:

$$
k_{r}=\frac{1}{\Delta}\left( \pm \arccos \left(1-\frac{\Delta^{2} k_{N}^{2}}{2}\right)+p 2 \pi\right), \quad p \in \mathbb{Z}
$$

Moreover, the existence of these roots is conditioned by

$$
\left(1-\frac{\Delta^{2} k_{N}^{2}}{2}\right)>-1
$$

which corresponds to the condition:

$$
n>\pi \text {. }
$$

This condition can be observed in figure 1 , where $E^{1 D}$ presents a zero only for the values of $n$ above $\pi$.

In order to delete singularities effects, it is proposed to introduce a correcting factor to $\kappa_{1 D}^{4}$. This factor, noted $\mu^{4}$, is defined by:

$$
\tilde{\kappa}_{1 D}^{4}=\mu^{4} \kappa_{1 D}^{4}
$$


Replacing $\kappa_{1 D}^{4}$ by $\tilde{\kappa}_{1 D}^{4}$ in equation (7), the roots of the modified $\tilde{E}^{1 D}$ numerator satisfy the relationship:

$$
\cos \left(k_{r} \Delta\right)=1-\frac{\Delta^{2} k_{N}^{2}}{2 \mu^{2}}
$$

The value of $\mu$ allowing $k_{r}$ to be equal to $k_{N}$ is:

$$
\mu=\frac{\Delta k_{N}}{\sqrt{2-2 \cos \left(k_{N} \Delta\right)}} .
$$

The roots of the modified $\tilde{E}^{1 D}$ numerator are then:

$$
k_{r}= \pm k_{N}+\frac{p 2 \pi}{\Delta}, \quad p \in \mathbb{Z}
$$

or, with respect to $\alpha_{r}=k_{r} / k_{N}$ :

$$
\alpha_{r}= \pm 1+n p, \quad p \in \mathbb{Z}
$$

The corrected FAT (CFAT) estimation of the force distribution is finally given by:

$$
p^{C F A T}(x)=E I \mu^{4} \delta_{\Delta}^{4 x}(x)-\rho S \omega^{2} w(x) .
$$

The wavenumber response $\tilde{E}^{1 D}$ of this CFAT scheme is drawn in figure 2 . The

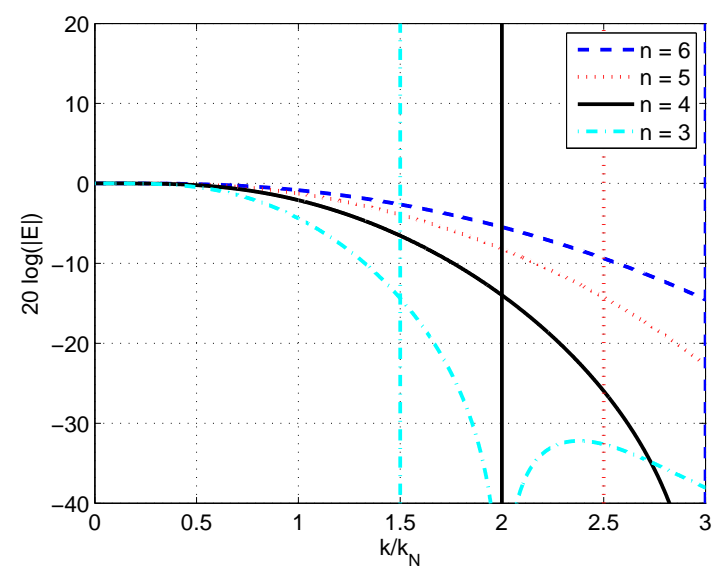

Fig. 2. Response of the corrected FAT identification in the wavenumber domain. Vertical lines indicate the Nyquist wavenumbers of the spatial sampling. 
low-pass filter is preserved, and the singularities are efficiently removed. It can be noted that secondary roots of $\tilde{E}^{1 D}$, given by equation (12) are also visible, at $\alpha=2$ for $n=3$ and $\alpha=3$ for $n=4$. The cut-off wavenumber of ideal anti-aliasing filters are also drawn for each value of $n$. The value of the CFAT responses at these wavenumbers is about $-15 \mathrm{~dB}$.

\subsection{High frequency limitation of the CFAT (Corrected FAT)}

The values of $n$ less than 3 points by natural wavelength correspond to the high frequency domain. The wavenumber responses of the CFAT in this domain are drawn in figure 3. A classic high frequency limit of FAT is based on the Shannon's sampling criterion for the natural wavelength of the beam (at least

2 points by natural wavelength). This is indeed the extreme limit if we are interested in the response of the beam. But this limitation does not concern the force distribution itself, whose wavenumber distribution is not depending on the structure.

It is interesting in observing figure 3 that decreasing the value of $n$ has two effects: the decrease of the cut-off wavenumber, and the increase of the energy of an out-of-phase secondary lobe in higher frequencies. This second effect induces aliasing which is dramatic because the energy of high wavenumber components (higher than the Nyquist limit, materialized by vertical lines in figure 3) is aliased on the identified load distribution. In this work, the limit is adjusted to keep this secondary lobe lower than $-10 \mathrm{~dB}$, which corresponds to $n>1.85$ (plain curve in figure 3 ). It can be noted that this limit is not acceptable when one considers the problem of the identification of the spatial response of the beam, because the Shannon's criterion is not satisfied for the 
natural wavenumber $(n>2)$. However, the force distribution can be studied up to a frequency corresponding to $n=1.85$, thanks to the anti-aliasing filter provided by the CFAT approach. $n$ being inversely proportional to the square root of the frequency, the high frequency limit can be increased by about $17 \%$, in comparison with the Shannon's criterion for the beam's response.

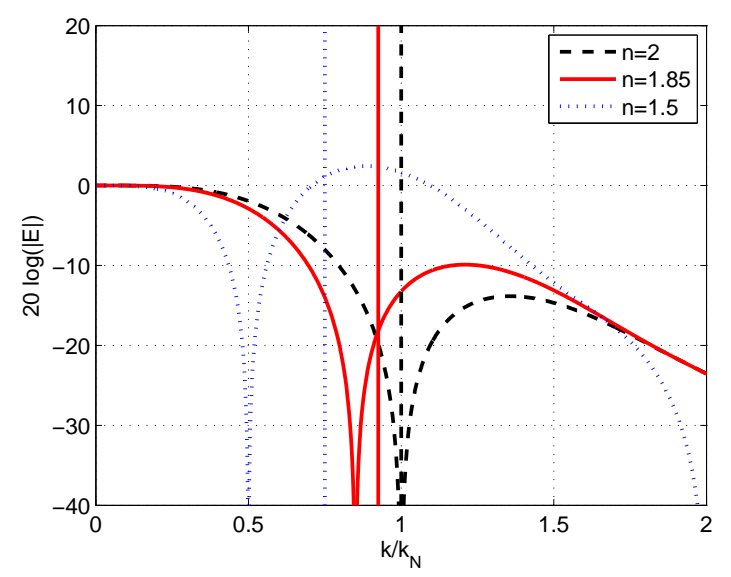

Fig. 3. Response of the corrected FAT identification in the wavenumber domain in high frequency (low values of $n$ ). Vertical lines indicate the Nyquist wavenumbers of the spatial sampling.

\subsection{Numerical illustrations for flexural beams}

Let us consider the analytic model of a simply supported beam. The displacement of the beam at the angular frequency $\omega$ excited by a $1 \mathrm{~N}$ (peak) force at $x_{e}$ is computed by the modal expansion:

$$
w(x)=\frac{2}{E I L} \sum_{i \geq 1} \frac{\sin \left(k_{i} x_{e}\right) \sin \left(k_{i} x\right)}{k_{i}^{4}-k_{N}^{4}}, \text { with } \quad k_{i}=\frac{i \pi}{L},
$$

and $L$ standing for the beam's length. Numerical values are written in table 1.

The high frequency limit of the simulation is fixed to 1.85 points by natural 


\begin{tabular}{|c|c|c|c|}
\hline Young's modulus & $E=210 e 9 \mathrm{~Pa}$ & density & $\rho=7800 \mathrm{~kg} / \mathrm{m}^{3}$ \\
\hline Damping & $\eta=1 \%$ & Length & $L=1 \mathrm{~m}$ \\
\hline thickness & $h=5 \mathrm{~mm}$ & Force position & $x_{e}=0.61 \mathrm{~m}$ \\
\hline space resolution & $\Delta=5 \mathrm{~cm}$ & Noise level & $\mathrm{SNR}=30 \mathrm{~dB}$ \\
\hline
\end{tabular}

Table 1

Numerical values of the beam used for the simulations.

wavelength (following the theoretical discussion in section 2.4), which corresponds to approximately $5.5 \mathrm{kHz}$. The modal truncation of the expansion (14) is chosen to keep modes with eigen frequencies up to 10 times the high frequency limit. For each measurement point, a white noise is added to computed velocities in order to satisfy a given signal to noise ratio equals to $30 \mathrm{~dB}$. The total number of simulated measurement points is 19 , from $0 \mathrm{~m}$ to $1 \mathrm{~m}$ with a sampling step of $0.05 \mathrm{~m}$.

Each point of the identified force distribution results from a centered difference scheme. That's why the force cannot be assessed at the two extreme points on both sides of the measurement mesh. The resulting force distribution is consequently assessed from $0.10 \mathrm{~m}$ to $0.9 \mathrm{~m}$ (called "identified force interval" in the following).

The results of FAT and CFAT are drawn in figure 4 with respect to the frequency, where force values are deduced from integrations over 2 points surrounding the input force location (at $x=0.6$ and $0.65 \mathrm{~m}$ ). It can be seen that the FAT and CFAT behaves similarly in low frequency $(<400 \mathrm{~Hz}, n>8)$, with strongly overestimated loads: this is the frequency range in which FAT needs to be regularized because of the noise amplification (see [6]). Between 400 and $1500 \mathrm{~Hz}(8>n>3.5)$, results are slightly different, but the result of classic 
FAT remains acceptable (error lower than $2 \mathrm{~dB})$. Above $1500 \mathrm{~Hz}(n<3.5)$, the CFAT error is still very small (less than $0.5 \mathrm{~dB}$ ) while the classic FAT error can reach 5 to $10 \mathrm{~dB}$. It can be noted that the CFAT result is still very good for $2>n>1.85$ (above the vertical black line), following theoretical expectations given in section 2.4 .

The reconstructed force distributions averaged between $500 \mathrm{~Hz}$ and $5.5 \mathrm{kHz}$ are

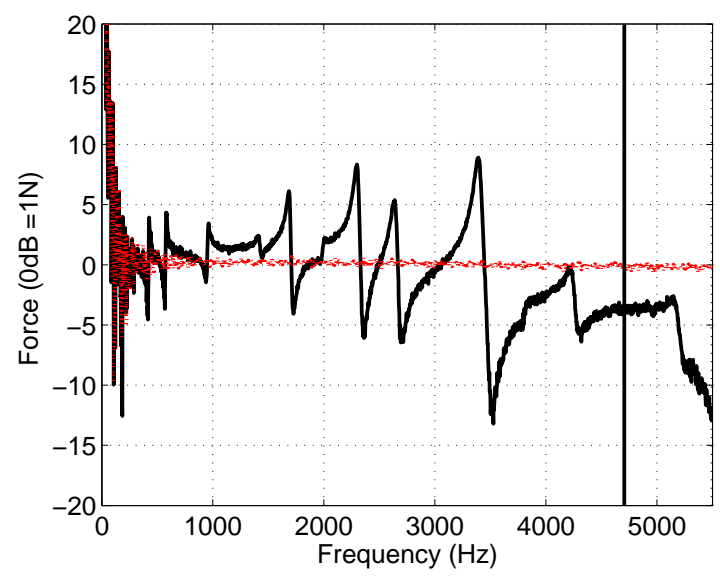

Fig. 4. Simulation results: identified force spectrum integrated over $x=0.60 \mathrm{~m}$ and $x=0.65 m$ using FAT (solid black) and FAT corrected (dotted red) . The injected value is $1 \mathrm{~N}(0 \mathrm{~dB})$ at each frequency step. The vertical black line corresponds to the resolution $n=2$ (the maximum frequency $5.5 \mathrm{kHz}$ corresponds to $n=1.85$ ).

plotted in figure 5. It is clear that the CFAT result is really good, the identified load is distributed exclusively over the two points of the grid surrounding the real input location $(x=0.6$ and $0.65 \mathrm{~m})$. The result of the classic FAT is not so satisfying: The load is almost localized, but slightly overestimated, and a residual load distribution is identified over the whole length of the beam.

A second simulation is carried out to assess the robustness of the method to changes in boundary conditions, and to more complex input force distributions. The same beam (same parameters than in table 1, same measurement mesh) is now considered as clamped at $x=0$ and free at $x=L$. The following 


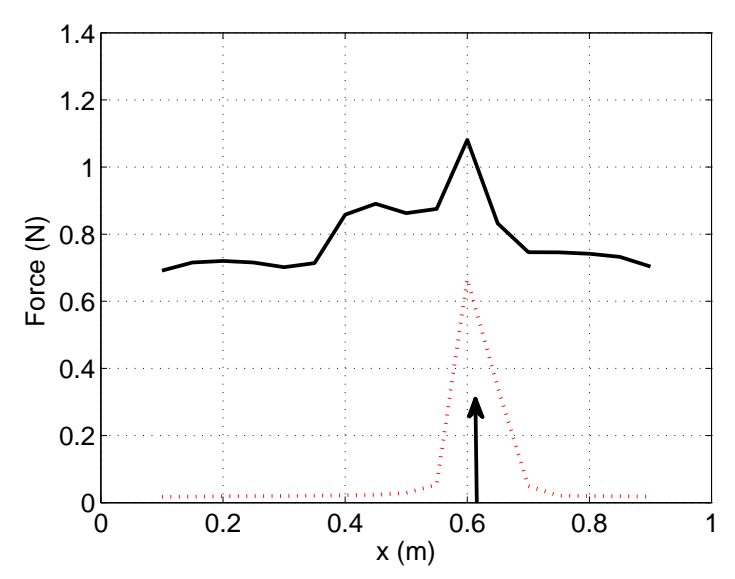

Fig. 5. Simulation results: identified force distributions using FAT (solid black) and CFAT (dotted red), averaged between 0.5 and $5.5 \mathrm{kHz}$. Vertical arrow : input force location $(1 \mathrm{~N}$ dirac at $x=0.61 m)$

modal decomposition is adopted to simulate measurements (from [17]):

$$
\begin{aligned}
w(x) & =\frac{1}{E I} \sum_{i \geq 1} \frac{1}{\left\|f_{i}(x)\right\|^{2}} \frac{f_{i}\left(x_{e}\right) f_{i}(x)}{k_{i}^{4}-k_{N}^{4}}, \\
\text { with } \quad f_{i}(x) & =\frac{\operatorname{ch}\left(k_{i} x\right)-\cos \left(k_{i} x\right)}{\operatorname{ch}\left(k_{i} L\right)+\cos \left(k_{i} L\right)}-\frac{\operatorname{sh}\left(k_{i} x\right)-\sin \left(k_{i} x\right)}{\operatorname{sh}\left(k_{i} L\right)+\sin \left(k_{i} L\right)}, \\
\text { and } \quad k_{1} L & =1.875, k_{2} L=4.694, k_{i} L=-\pi / 2+i \pi \text { for } i \geq 3 .
\end{aligned}
$$

The same force $(1 \mathrm{~N})$ is injected at the same location $x=0.61 \mathrm{~m}$, but two other ones (also $1 \mathrm{~N}$ ) are also injected, the first one at $x=0.15 \mathrm{~m}$ (inside the identified force interval 0.10 to $0.90 \mathrm{~m}$ ), and the second one at $x=1 \mathrm{~m}$, i.e. at the beam's free end (outside the identified force interval).

The identified force spectrum integrated over the two points surrounding the force location $(x=0.60 m$ and $x=0.65 m)$ is drawn in figure 6 . The same observations than for the simply supported case can be made : the identified force spectrum using CFAT is satisfying upon $500 \mathrm{~Hz}$, while the FAT results is very flawed above $1500 \mathrm{~Hz}$.

The results are drawn in the space domain in figure 7 , averaged between 0.5 
and $5.5 \mathrm{kHz}$. The CFAT results allows clearly the localization of input forces that are in the identified force interval. The force injected at the free end of the beam does not prevent neither the localization nor the quantification of other forces. Results using FAT are very noisy, input forces cannot be clearly localized in figure 7.

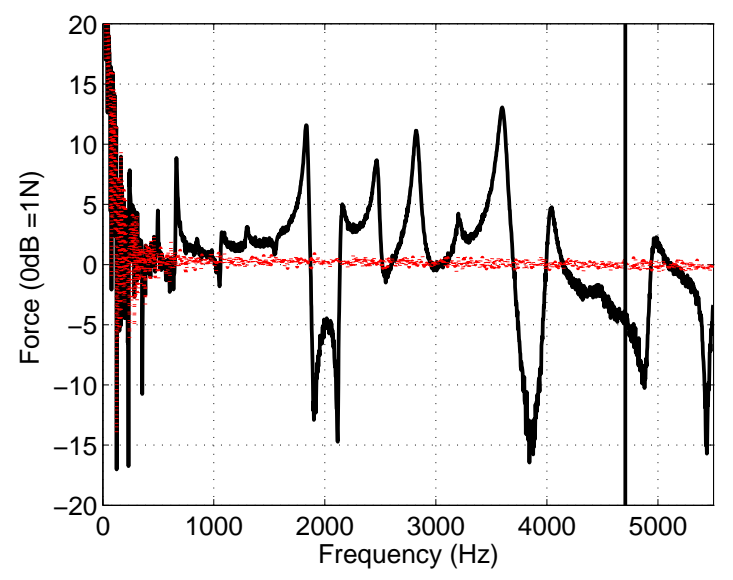

Fig. 6. Clamped-free simulation. Identified force spectrum integrated over $x=0.60 \mathrm{~m}$ and $x=0.65 m$ using FAT (solid black) and FAT corrected (dotted red). The injected value is $1 \mathrm{~N}(0 \mathrm{~dB})$ at each frequency step. The vertical black line corresponds to the resolution $n=2$ (the maximum frequency $5.5 \mathrm{kHz}$ corresponds to $n=1.85$ ).

\section{Identification of load distributions on plates}

\subsection{Classic FAT for flexural plates}

The motion equation of flexural plates at the angular frequency $\omega$ is:

$$
D\left(\frac{\partial^{4} w}{\partial x^{4}}+\frac{\partial^{4} w}{\partial y^{4}}+2 \frac{\partial^{4} w}{\partial x^{2} \partial y^{2}}\right)-\rho h \omega^{2} w(x, y)=p(x, y)
$$




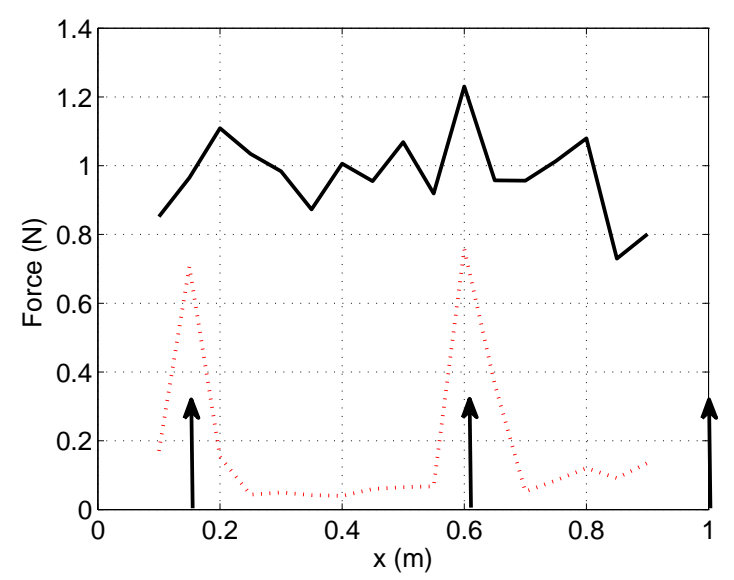

Fig. 7. Clamped-free simulation. Identified force distributions using FAT (solid black) and CFAT (dotted red), averaged between 0.5 and $5.5 \mathrm{kHz}$. Vertical arrows : input force locations ( $1 \mathrm{~N}$ diracs at $x=0.61 \mathrm{~m}, x=0.15 \mathrm{~m}$ and $x=1 \mathrm{~m}$ )

where $D=E h^{3}\left(12\left(1-\nu^{2}\right)\right)^{-1}$ is the flexural stiffness per unit length, $\rho h$ is the mass per unit area, $p(x, y)$ is the load distribution in $N / m^{2}$ and $w(x, y)$ is the displacement in $m$.

A finite difference approximation with 13 points is used to assess the fourth derivatives of the displacement. $\left(\partial^{4} w / \partial x^{4}\right)$ and $\left(\partial^{4} w / \partial y^{4}\right)$ are approximated as in eq. 2 , whereas $\left(\partial^{4} w / \partial x^{2} \partial y^{2}\right)$ is computed as follows :

$$
\begin{aligned}
\frac{\partial^{4} w}{\partial x^{2} \partial y^{2}} \approx & \delta_{\Delta}^{2 x 2 y} \\
\approx & \frac{1}{\Delta^{4}} \sum_{p=-1}^{1} \sum_{q=-1}^{1} \psi_{p q} w(x+p \Delta, y+q \Delta) \\
\text { with } \quad & \psi_{00}=4 \\
& \psi_{-10}=\psi_{10}=\psi_{0-1}=\psi_{01}=-2 \\
& \psi_{-1-1}=\psi_{11}=\psi_{1-1}=\psi_{-11}=1
\end{aligned}
$$

The FAT estimation of the load distribution at the point coordinates $(x, y)$ is 


$$
\begin{aligned}
p^{F A T}(x, y)= & D \Phi_{\Delta}(x, y)-\rho S \omega^{2} w(x, y) \\
\text { with } & \Phi_{\Delta}(x, y)=\delta_{\Delta}^{4 x}+\delta_{\Delta}^{4 y}+2 \delta_{\Delta}^{2 x 2 y}
\end{aligned}
$$

\section{2 $2 D$ wavenumber response of FAT for flexural plates}

The 2D Fourier transform of equation (16) is:

$$
\left(D\left(k_{x}^{2}+k_{y}^{2}\right)^{2}-\rho h \omega^{2}\right) \hat{w}\left(k_{x}, k_{y}\right)=\hat{p}\left(k_{x}, k_{y}\right)
$$

The 2D Fourier transform of the finite difference approximation $\Phi_{\Delta}(x, y)$ in eq. (18) is:

$$
\begin{aligned}
\hat{\Phi}_{\Delta}\left(k_{x}, k_{y}\right)=\frac{\hat{w}\left(k_{x}, k_{y}\right)}{\Delta^{4}}[ & 2 \cos \left(2 k_{x} \Delta\right)-8 \cos \left(k_{x} \Delta\right)+6 \\
& +2 \cos \left(2 k_{y} \Delta\right)-8 \cos \left(k_{y} \Delta\right)+6 \\
& +\cos \left(\left(k_{x}+k_{y}\right) \Delta\right)+\cos \left(\left(k_{x}-k_{y}\right) \Delta\right) \\
& \left.+4-4 \cos \left(k_{x} \Delta\right)-4 \cos \left(k_{y} \Delta\right)\right],
\end{aligned}
$$

that can be simplified by:

$$
\begin{aligned}
\hat{\Phi}_{\Delta}\left(k_{x}, k_{y}\right)= & \hat{w}\left(k_{x}, k_{y}\right) \kappa_{2 D}^{4} \\
\text { with } \quad \kappa_{2 D}^{4}= & \frac{4(1-X)^{2}+4(1-Y)^{2}+8(1-X)(1-Y)}{\Delta^{4}}, \\
& X=\cos \left(k_{x} \Delta\right), Y=\cos \left(k_{y} \Delta\right) .
\end{aligned}
$$

The FAT estimation of the load distribution in the 2D wavenumber domain is thus:

$$
\hat{p}^{F A T}\left(k_{x}, k_{y}\right)=\hat{w}\left(k_{x}, k_{y}\right)\left(D \kappa_{2 D}^{4}-\rho h \omega^{2}\right) .
$$

The response of FAT is the ratio between real and identified load distributions:

$$
E^{2 D}\left(\omega, \Delta, k_{x}, k_{y}\right)=\frac{\hat{p}^{F A T}\left(k_{x}, k_{y}\right)}{\hat{p}\left(k_{x}, k_{y}\right)}=\frac{\kappa_{2 D}^{4}-k_{N}^{4}}{\left(k_{x}^{2}+k_{y}^{2}\right)^{2}-k_{N}^{4}}
$$


where $k_{N}$ is the natural flexural wavenumber of the plate

$$
k_{N}=\sqrt[4]{\frac{\rho h}{D} \omega^{2}} .
$$

\subsection{Correction of FAT for flexural plates}

The response of FAT, defined in eq. (22), has a singularity for $k_{x}^{2}+k_{y}^{2}=k_{N}^{2}$, which corresponds to a circle equation in the wavenumber domain. As for the 1D case, it is proposed to modify the finite difference approximation by the introduction of two constants $\mu$ and $\nu$, as follows:

$$
\tilde{\Phi}_{\Delta}(x, y)=\mu^{4}\left(\delta_{\Delta}^{4 x}+\delta_{\Delta}^{4 y}\right)+2 \nu^{4} \delta_{\Delta}^{2 x 2 y} .
$$

The 2D Fourier Transform of eq. (23) gives:

$$
\begin{aligned}
& \hat{\tilde{\Phi}}_{\Delta}\left(k_{x}, k_{y}\right)= \hat{w}\left(k_{x}, k_{y}\right) \tilde{\kappa}_{2 D}^{4}, \\
& \text { with } \quad \tilde{\kappa}_{2 D}^{4}=\frac{4 \mu^{4}(1-X)^{2}+4 \mu^{4}(1-Y)^{2}+8 \nu^{4}(1-X)(1-Y)}{\Delta^{4}} \\
& \quad X=\cos \left(k_{x} \Delta\right), Y=\cos \left(k_{y} \Delta\right) .
\end{aligned}
$$

The value of $\mu$ is chosen to equalize the roots of the numerator and the denominator of equation $(22)$ (replacing $\kappa_{2 D}^{4}$ by $\left.\tilde{\kappa}_{2 D}^{4}\right)$ for $k_{x}=0(X=1$ and $\left.k_{y}=k_{N}\right)$ or $k_{y}=0\left(Y=1\right.$ and $\left.k_{x}=k_{N}\right)$. The result is:

$$
\mu^{4}=\frac{\Delta^{4} k_{N}^{4}}{4\left[1-\cos \left(k_{N} \Delta\right)\right]^{2}},
$$

which corresponds exactly to the correction for the 1D case.

The value of $\nu$ is determined by equalizing roots of the numerator and denominator of equation (22) for $k_{x}=k_{y}=k_{N} / \sqrt{2}$ which leads to: 


$$
\nu^{4}=\frac{\Delta^{4} k_{N}^{4}}{8\left[1-\cos \left(\frac{k_{N} \Delta}{\sqrt{2}}\right)\right]^{2}}-\mu^{4}
$$

The CFAT estimation of the load is finally expressed as follows:

$$
p^{C F A T}(x, y)=D\left(\mu^{4} \delta_{\Delta}^{4 x}+\mu^{4} \delta_{\Delta}^{4 y}+2 \nu^{4} \delta_{\Delta}^{2 x 2 y}\right)-\rho S \omega^{2} w(x, y) .
$$

The wavenumber responses of the classical and corrected FAT are drawn in figure 8 for $n=5$ and $n=3$ ( $n$ being the number of points by natural wavelength). The Nyquist circle of the spatial sampling is materialized (dashed line), representing the cutoff wavenumber of an ideal anti-aliasing filter for the load sampling. Both FAT and CFAT keep their inherent low-pass filtering effect. The singularity circle is clearly observed on the classic FAT response at $k_{x}^{2}+k_{y}^{2}=k_{N}^{2}$. The correction attenuates efficiently this singularity, but contrary to the $1 \mathrm{D}$ correction, the singularity is not completely deleted. For $n=3$, the response is continuous only on the points of the singularity circle corresponding to $\left|k_{x}\right|=0,\left|k_{y}\right|=0, k_{x}=k_{y}=k_{N} / \sqrt{2}$, which is imposed by the correction terms. However, a short singularity is still observed outside these points. This singularity is also present for higher values of $n$, but is smaller and is not visible on the $2 \mathrm{D}$ representations, as it is the case for $n=5$.

\subsection{High frequency limitation of the corrected FAT for flexural plates}

In order to study the high frequency limit of the method, it is proposed here to see what is the acceptable smallest values of $n$. For the beam the limit was fixed to $n=1.85$ (secondary lobe of the wavenumber response lower than -10 

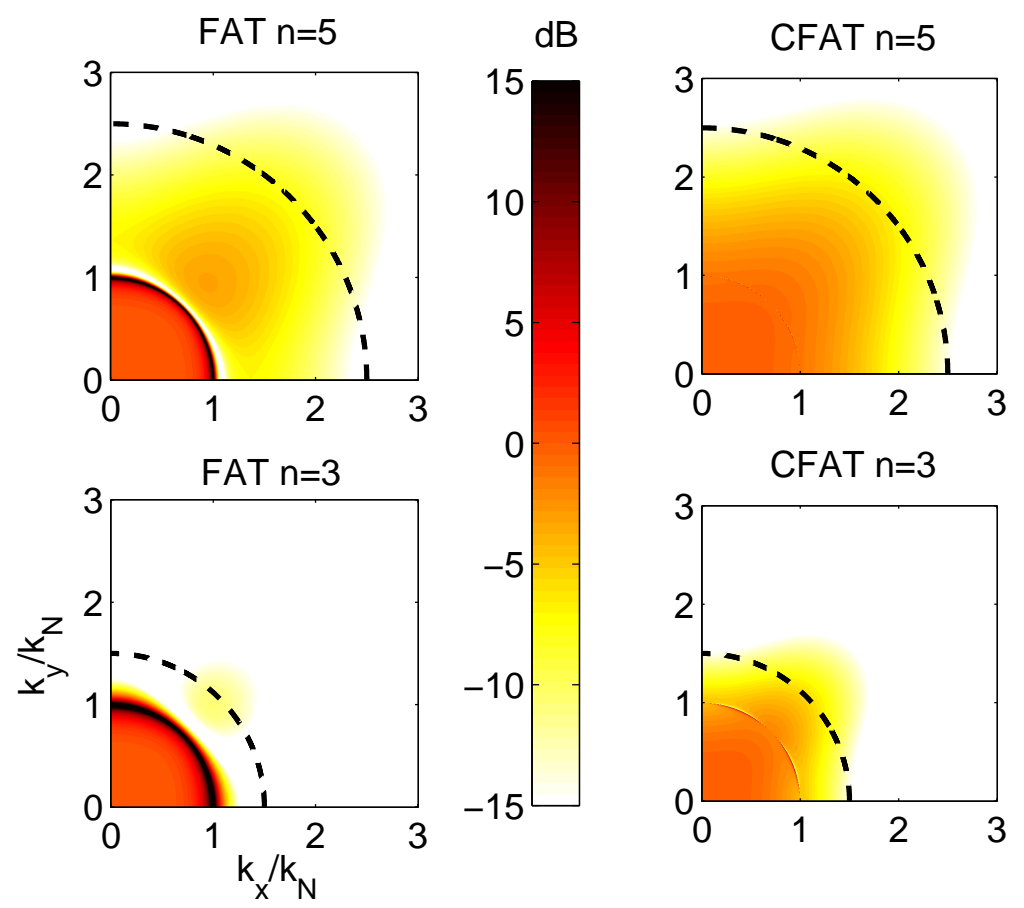

Fig. 8. Wavenumber responses of FAT (left) and CFAT (right) for $n=5$ (top) and $n=3$ (bottom) ( $n$ is the ratio $\lambda_{N} / \Delta$ ). Dashed black line: Nyquist circle of the spatial sampling.

$\mathrm{dB}$ ). The $2 \mathrm{D}$ wavenumber response of the CFAT for plates is drawn in figure 9 for $n=2$ and for $n=1.85$. The singularity is not efficiently smoothed for low values of $\mathrm{n}$. For the case $n=1.85$, this remaining singularity induces sharp and strong amplifications, at wavenumbers upon the Nyquist circle. Thus the limit for the 2D case should be kept for $n>2$. 

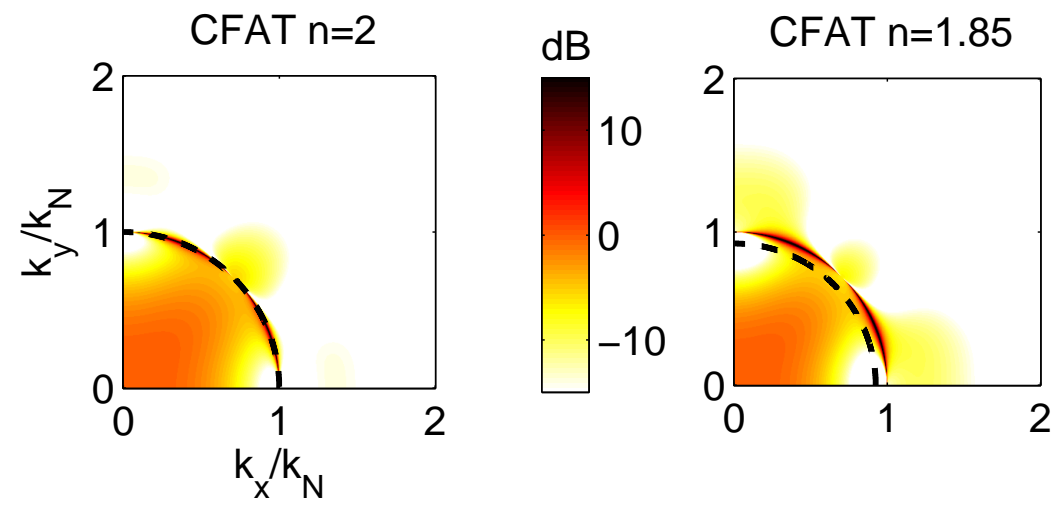

Fig. 9. Wavenumber responses of CFAT for $n=2$ (left) and $n=1.85$ (right). Dashed black line: Nyquist circle of the spatial sampling. 


\subsection{Numerical illustration for flexural plates}

The analytic model for the simply supported flexural rectangular plate is considered here. The displacement of the plate excited by a unitary point force at the coordinates $\left(x_{e}, y_{e}\right)$, for the angular frequency $\omega$, is computed by the modal expansion:

$$
\begin{aligned}
w(x, y)= & \frac{4}{D L_{x} L_{y}} \sum_{i, j \geq 1} \frac{\sin \left(k_{i} x_{e}\right) \sin \left(k_{j} y_{e}\right) \sin \left(k_{i} x\right) \sin \left(k_{j} y\right)}{\left(k_{i}^{2}+k_{j}^{2}\right)^{2}-k_{N}^{4}}, \\
& \text { with } k_{i}=\frac{i \pi}{L x}, k_{j}=\frac{j \pi}{L y}
\end{aligned}
$$

$L_{x}$ and $L_{y}$ standing for the plate's dimensions. Numerical values used for the simulations are given in table 2 .

\begin{tabular}{|c|c|c|c|}
\hline Young's modulus & $E=72 e 9 P a$ & density & $\rho=2700 \mathrm{~kg} / \mathrm{m}^{3}$ \\
\hline Damping & $\eta=1 \%$ & Dimensions & $L_{x} \times L_{y}=0.7 \times 0.5 \mathrm{~m}^{2}$ \\
\hline thickness & $h=5 \mathrm{~mm}$ & Force location & $\left(x_{e}, y_{e}\right)=(0.31,0.21) \mathrm{m}$ \\
\hline space resolution & $\Delta=3 \mathrm{~cm}$ & Noise level & $\mathrm{SNR}=30 \mathrm{~dB}$ \\
\hline
\end{tabular}

Table 2

Numerical values for the plate simulation.

The high frequency limit of the simulation is fixed to ensure a minimum of 2 points by natural wavelength, which corresponds to $8 \mathrm{kHz}$ in this simulation. The modal summation in equation (25) is truncated to keep modes with eigen frequencies up to 10 times the high frequency limit. Some noise is also added to simulate uncertainties in the measurements. This is made by the addition of a white noise to satisfy a signal to noise ratio of $30 \mathrm{~dB}$. 
Results of FAT and CFAT are drawn in figure 10 with respect to the frequency. The result is integrated over 4 points surrounding the input force location. Below $500 \mathrm{~Hz}(n>8)$, noise dominates because the spacing is small and the finite difference schemes cannot regularize the problem (no filtering effect). Between $500 \mathrm{~Hz}$ and $1300 \mathrm{~Hz}(5<n<8)$, FAT and CFAT give results with errors less than $5 \mathrm{~dB}$. Above $1300 \mathrm{~Hz}$, CFAT becomes highly accurate, the errors are less than $1 \mathrm{~dB}$, whereas FAT results are strongly biased with errors that can reach more than $10 \mathrm{~dB}$.

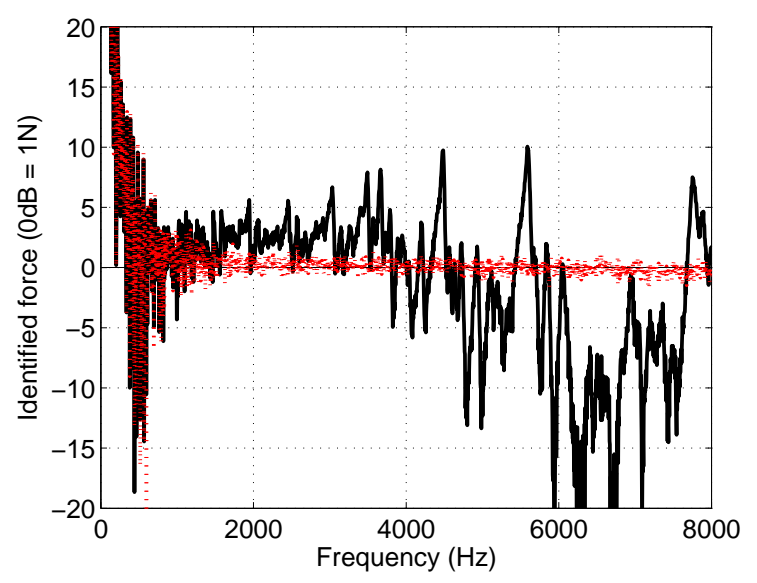

Fig. 10. Simulation results: identified force spectrum using FAT (black) and FAT corrected (dotted red)

Results of FAT and CFAT are drawn in figure 11 with respect to $x$ and $y$, where the force levels are integrated between 1 and $8 \mathrm{kHz}$. The input force is correctly localized with FAT or CFAT, but the level of erroneous loads identified on the whole surface of the plate is much lower with CFAT $(\approx-25 d B)$ than with $\operatorname{FAT}(\approx-5 d B)$ 

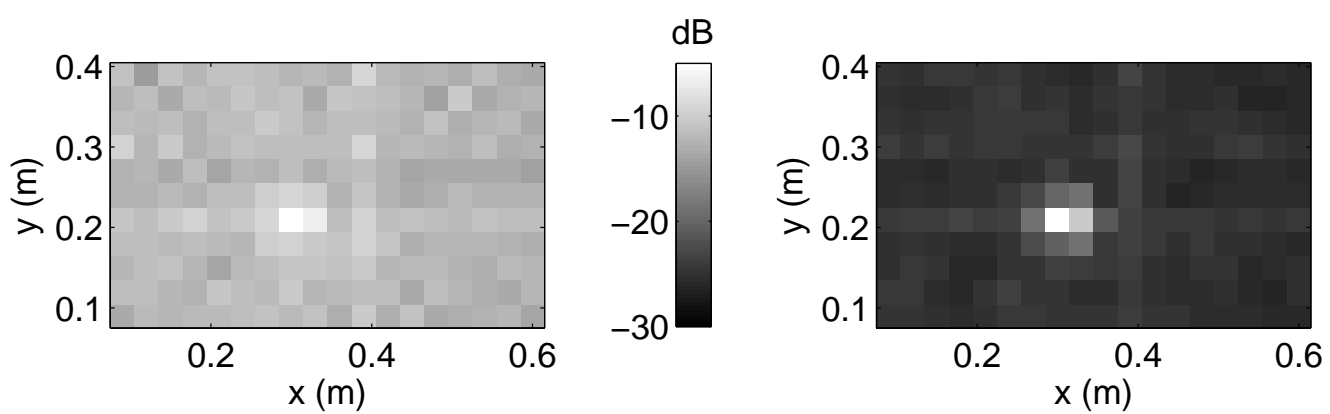

Fig. 11. Simulation results: identified force distributions using FAT (left) and CFAT (right) overall levels between 1 and $8 \mathrm{kHz}$. Both maps are drawn using a 25dB dynamic range.

\section{Conclusion}

The main role of the finite difference schemes in FAT is to approximate the displacement derivatives in the equation of motion. Usually, in the classic FAT, the spacing between sensors is chosen to be small in order to have a good assessment of the derivatives and a wavenumber filter is used to eliminate noises intrinsically amplified by the derivatives. In this study, it is shown that the schemes also constitute low-pass wavenumber filters, which may be used for the regularisation of FAT if larger spacings are chosen. To do so, a correction must be made and amplification terms are proposed in this paper. Simulations on beams and plates show that it is possible to use the FAT with only the required number of points for the finite difference schemes, i.e. five or thirteen points for the beam or the plate, respectively.

\section{References}

[1] F.D. Bartlett and W.G. Flannelly. Model verification of force determination for measuring vibratory loads. Journal of the American Helicopter Society, 
24:10-18, 1979.

[2] B. Hillary and D.J. Ewins. The use of strain gauges in force determination and frequency response function measurements. In Proceedings of IMAC 2, Orlando, FL, USA, 1984.

[3] K.S. Stevens. Force identification problems-an overview. In Proceedings of SEM Spring Conference on Experimental Mechanics, pages 838-844, Houston, TX, USA, 1987.

[4] W. Hendricx and D. Vandenbroek. Suspension analysis in view of road noise optimization. S.A.E. Technical paper series, (931343), 1993.

[5] P. Mas, P. Sas, and K. Wyckaert. Indirect force identification based upon impedance matrix inversion : a study on statistical and deterministical accuracy. In Proceedings of ISMA 19, Leuven, Belgium, September 1994.

[6] C. Pezerat and J.-L. Guyader. Two inverse methods for localization of external sources exciting a beam. Acta Acustica, 3:1-10, 1995.

[7] C. Pezerat and J.-L. Guyader. Force analysis technique: Reconstruction of force distribution on plates. Acustica united with Acta Acustica, 86:322-332, 2000.

[8] M.S. Djamaa, N. Ouella, C. Pezerat, and J-L. Guyader. Reconstruction of a distributed force applied on a thin cylindrical shell by an inverse method and spatial filtering. Journal of Sound and Vibration, 301:560-575, 2007.

[9] D.U. Noiseux. Measurement of power flow in uniform beams and plates. Journal of the Acoustical Society of America, 47(1 (Part 2)):238-247, 1969.

[10] G. Pavic. Measurement of structure borne wave intensity, part 1 : Formulation of the methods. Journal of the Acoustical Society of America, 49(2):221-230, 1976. 
[11] E. G. Williams, H. D. Dardy, and R. D. Fink. A technique for measurement of structure-borne intensity in plates. Journal of the Acoustical Society of America, 78(6):2061-2068, 1985.

[12] Y. Zhang and J.A. Mann III. Measuring the structural intensity and force distribution in plates. Journal of the Acoustical Society of America, 99(1):345$353,1996$.

[13] A. Fregolent and A. Sestieri. Force identification from vibration measurements in the wavenumber domain. In Proceedings of ISMA 21, Leuven, Belgium, 1996.

[14] Q. Leclere and C. Pezerat. Time domain identification of loads on platelike structures using an array of acoustic velocity sensors. In proceedings of Acoustics08, Paris, France, 2008.

[15] C. Pezerat, T. Loyau, and J.-L. Guyader. Characterisation of vibration sources on a set of plates using the riff technique. Noise Control Engineering Journal, 50(2):50-57, MAR-APR 2002.

[16] C. Pezerat, Q. Leclere, N. Totaro, and M. Pachebat. Identification of vibration excitations from acoustic measurements using near field acoustic holography (nah) and the force analysis technique (fat). Journal of Sound and Vibration, 326:540-556, 2009.

[17] J.-L. Guyader. Vibration in continuous media. ISTE, 2006. 